\title{
Revista Colombiana de

\section{Capítulo 6. Utilidad del mapeo tridimensional en la ablación de la fibrilación auricular paroxística}

\section{Chapter 6. Usefulness of three-dimensional mapping in paroxysmal atrial fibrillation}

\author{
Alberto Negrete ${ }^{\mathrm{a}, \mathrm{b}, *}$ y Efraín Gil ${ }^{\mathrm{a}, \mathrm{b}}$ \\ ${ }^{a}$ Centro Médico Imbanaco, Cali, Colombia

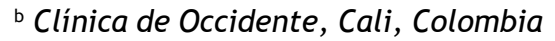

Recibido el 16 de noviembre de 2015; aceptado el 18 de enero de 2016

\section{Introducción}

La fibrilación auricular es la arritmia sostenida más común y genera aumento en la morbimortalidad de los pacientes que la padecen ${ }^{1}$. Clásicamente se ha clasificado como paroxística, persistente y permanente; sin embargo, no hay una definición uniforme de fibrilación auricular paroxística. Para las sociedades estadounidenses de cardiología y arritmias se considera como aquella que espontáneamente o con manejo médico pasa a ritmo sinusal dentro de los siete días del inicio de la arritmia, usualmente en las primeras 48 horas $^{2}$. No obstante, para la Sociedad Europea de Cardiología la definición es levemente diferente ya que solo acepta como fibrilación auricular paroxística aquella que espontáneamente pasa a ritmo sinusal en menos de siete días ${ }^{3}$.

Independiente de la definición, la carga de fibrilación auricular ha estado incrementándose constantemente, y hoy se estima que más de 2 millones de estadounidenses y 4 millones de europeos se ven afectados ${ }^{4}$.

De otro lado, el tratamiento de estos pacientes se enfoca en cuatro pilares: manejo de la patología cardiovascular subyacente y los factores de riesgo, prevención de tromboembolia, control de la frecuencia cardiaca y restauración o mante- nimiento del ritmo sinusal (estrategia de control del ritmo), en un subgrupo de pacientes. En este último pilar las estrategias se reducen al uso de antiarrítmicos o a la ablación por radiofrecuencia.

Los antiarrítmicos han tenido una eficacia no ideal o se han acompañado de múltiples efectos adversos. En un metaanálisis llevado a cabo en 2006 en el que se incluyeron 3.180 pacientes y se enfocaron en cinco antiarrítmicos (amiodarona, propafenona, sotalol, dofetilide y flecainide) se encontró un porcentaje de éxito (definido como desaparición de la arritmia) de 52 (intervalo de confianza del 95\%, 47-57\%) ${ }^{5}$. De acuerdo con ello, un grupo importante de pacientes no mejoran con el manejo médico antiarrítmico.

Por tanto, la mayoría de estudios de eficacia de la ablación en la fibrilación auricular paroxística se han realizado en pacientes refractarios al manejo médico antiarrítmico. Un metaanálisis reciente encontró que en este grupo el éxito es del $77,8 \%$ frente a $23,4 \%$ con manejo antiarrímico ${ }^{6}$. La mayoría de estos pacientes tenían corazón sano y eran relativamente jóvenes (edad promedio de 57 años). Con base en esta evidencia, las guías recomiendan como clase I con nivel de evidencia A la ablación en fibrilación auricular paroxística en aquellos pacientes refractarios al manejo antiarrítmico ${ }^{2,3}$. Sin

*Autor para correspondencia.

Correo electrónico: alberto.negrete@imbanaco.com.co (A. Negrete). 
embargo, los registros mundiales con más de 9.000 pacientes muestran menor frecuencia de eficacia (alrededor del $52 \%)^{7}$. Por otra parte, los seguimientos a largo plazo (5 años) reportan recurrencias entre el 25 y $71 \%$, por lo que es evidente que por ahora no se considere un procedimiento curativo y se utilice el término, "paciente libre de arritmia"8-10.

Por otra parte, se ha investigado si la ablación puede ser la primera línea de terapia, antes de los antiarrítmicos, en pacientes con fibrilación auricular paroxística. El estudio RAAFT, con 127 pacientes, fue el primero que encontró beneficio en este grupo de pacientes con ausencia de eventos a un año en el $48 \%$ de pacientes con ablación y $28 \%$ en aquellos con manejo antiarrímico $(p=0,02)^{11}$. Sin embargo el estudio MANTRA-PAF en 294 pacientes, con seguimiento a dos años, mostró menores eventos sintomáticos en el grupo de ablación aunque sin diferencias en la carga de fibrilación auricular en los dos grupos de tratamiento ${ }^{12}$. Las guías internacionales dan recomendación lla con nivel de evidencia $B$ a la terapia de ablación como manejo inicial (terapia de primera línea) en pacientes seleccionados con fibrilación auricular paroxística.

\section{Utilidad del mapeo tridimensional}

Aunque no hay un acuerdo universal acerca de cuál es la técnica ideal de ablación en el manejo del paciente con fibrilación auricular paroxística, todas incluyen el aislamiento de venas pulmonares. El aislamiento antral de las venas pulmonares ha surgido como alternativa óptima dado que un número significativo de episodios de fibrilación auricular se inicia en el área de dichas venas ${ }^{13,14}$ (fig. 1). La efectividad de esta técnica en estos pacientes es de un 70 a $90 \%$, aunque en un 10 a $25 \%$ se requiere una segunda intervención. Cuanto más antral se lleve a cabo el aislamiento de las venas pulmonares, mejor, ya que se incluirá en él una mayor cantidad de tejido potencialmente arritmogénico, existiendo además menor probabilidad de estenosis ${ }^{15,16}$.

El seguimiento a largo plazo muestra que en pacientes con recurrencia de fibrilación auricular después del aislamiento de las venas pulmonares, la reconexión es muy común. Un

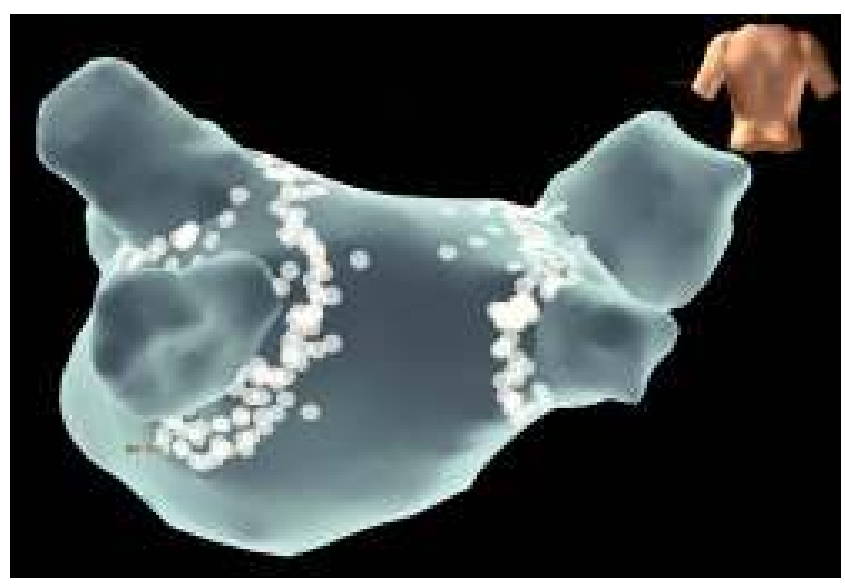

Figura 1 Imagen de las lesiones de radiofrecuencia en el aislamiento de venas pulmonares (puntos en blanco alrededor de los antros). Se muestra una reconstrucción 3-D de la aurícula izquierda fusionada con una imagen tomográfica tomada el día anterior (vista posterior). estudio con 161 pacientes con seguimiento a 5 años encontró recurrencia del 47\%. En 66 pacientes se repitió el procedimiento y se encontró reconexión de las venas pulmonares en el $94 \%{ }^{17}$. Estos datos junto a otros estudios han llevado a postular que la reconexión eléctrica de las venas pulmonares es un mecanismo importante asociado a la recurrencia.

Se han considerado varios mecanismos responsables de la reconexión. Primero, es posible que el procedimiento inicial haya fallado en alcanzar el aislamiento, permitiendo la presencia de brechas o "gaps" residuales dentro de la lesión circular o que no haya sido posible lograr que las lesiones fueran transmurales ${ }^{18}$. En estos pacientes la recurrencia podría ser la expresión de un procedimiento incompleto de aislamiento de las venas pulmonares. Otros mecanismos postulados incluyen inflamación postablación o recaídas no relacionadas con reconexión de las venas pulmonares, sino con activación de otros "disparadores" localizados en otras venas cardiacas (vena cava superior, ligamento de Marshall o seno coronario $)^{19,20}$.

Para obtener una lesión continua es importante la ayuda del mapeo tridimensional (3-D), que permite localizar cada lesión y observar la continuidad de cada una de ellas para formar las líneas que llevan al aislamiento eléctrico de las venas pulmonares. Aunque no hay estudios aleatorizados que comparen la ablación guiada por fluoroscopia versus la guiada por mapeo 3-D es claro que esta última proporciona mayor fidelidad de la localización de las lesiones y menor exposición del paciente y el personal médico y paramédico de la sala a los rayos $X$. Los sistemas de obtención de mapas 3-D y los nuevos catéteres con tecnología de contacto están facilitando la ablación, mejorando su eficacia y su seguridad ${ }^{21}$. El uso de sistemas de navegación tridimensional optimiza el procedimiento de ablación al proporcionar una imagen virtual 3-D de la aurícula izquierda, permitiendo correlacionar la posición del catéter con la anatomía tridimensional (y no únicamente con la imagen radiológica bidimensional ${ }^{22}$. Por otro lado, la posibilidad de fusión de la imagen 3-D obtenida mediante el mapeo electroanatómico con la de una resonancia magnética (RM) o tomografía computarizada (TC) realizadas antes del procedimiento, facilita la identificación de anomalías de las venas pulmonares o anatomías auriculares izquierdas complejas. Sin embargo, estos sistemas todavía presentan algunas limitaciones. Es fundamental un mapeo cuidadoso y detallado de la aurícula izquierda y su fusión con las imágenes obtenidas mediante RM/TC para garantizar una buena concordancia entre la figura y la anatomía real del paciente. Por otra parte, el movimiento del paciente o los cambios de impedancia intratorácica pueden provocar un desplazamiento de la figura, de ahí que sea preciso repetir el mapa de la aurícula izquierda. Es imperativo comprobar la posición del catéter en relación con la posición de las venas pulmonares u orejuela izquierda durante todo el procedimiento ${ }^{23,24}$.

Los sistemas de mapeo 3-D han desarrollado aplicaciones complementarias de tecnología de contacto (Contact Force para el sistema CARTO y Tacticath para el sistema EnSite) $)^{25,26}$ que permiten establecer en tiempo real la fuerza de contacto entre la punta del catéter y la superficie cardiaca, lo cual es determinante en la eficacia y, particularmente, en la seguridad de los procedimientos, ya que permite adaptar los parámetros físicos de la aplicación de radiofrecuencia al gramaje 
de la fuerza de contacto reportada por el sistema. Está claramente definido que aplicaciones con menos de 10 gramos de presión son ineficaces y determinan recurrencias, y que aquellas con más de 40 gramos de presión incrementan dramáticamente la tasa de perforaciones y taponamiento cardiaco. Los estudios han demostrado impacto de esta tecnología en la eficacia, la seguridad y los tiempos del procedimiento, así como reducción en la exposición a radiación ionizante.

\section{Recomendaciones}

\section{Clase I}

- El uso de los sistemas de mapeo tridimensional para la ablación de fibrilación auricular paroxística está indicado. El uso de ecografía intracardiaca y catéteres de fuerza de contacto puede optimizar los resultados en términos de seguridad y eficacia (nivel de evidencia C).

\section{Bibliografía}

1. Go AS, Hylek EM, Phillips KA, et al. Prevalence of diagnosed atrial fibrillation in adults: national implications for rhythm management and stroke prevention: the AnTicoagulation and Risk Factors in Atrial Fibrillation (ATRIA) Study. JAMA. 2001;285:2370-5.

2. January CT, Wann LS, Alpert JS, Calkins H, et al. 2014 AHA/ACC/ HRS Guideline for the Management of Patients With Atrial Fibrillation A Report of the American College of Cardiology/American Heart Association Task Force on Practice Guidelines and the Heart Rhythm Society Developed in Collaboration With the Society of Thoracic Surgeons. Circulation. 2014;130:e199-267.

3. Camm J, Kirchhof P, Lip J, et al. Guidelines for the management of atrial fibrillation The Task Force for the Management of Atrial Fibrillation of the European Society of Cardiology (ESC). Eur Heart J. 2010;31:2369-429.

4. Miyasaka Y, Barnes ME, Gersh BJ, Cha SS, et al. Secular trends in incidence of atrial fibrillation in Olmsted County, Minnesota, 1980 to 2000 , and implications on the projections for future prevalence. Circulation. 2006;114:119-25.

5. Calkins H, Reynolds MR, Spector P, Sondhi M, Xu Y, Martin A, et al. Treatment of atrial fibrillation with antiarrhythmic drugs or radiofrequency ablation: two systematic literature reviews and meta-analyses. Circ Arrhythm Electrophysiol. 2009;2:349-61.

6. Bonanno C, Paccanaro M, La Vecchia L, Ometto R, Fontanelli A. Efficacy and safety of catheter ablation versus antiarrhythmic drugs for atrial fibrillation: a meta-analysis of randomized trials. J Cardiovasc Med (Hagerstown). 2010;11:408-18.

7. Cappato R, Calkins H, Chen SA, et al. Worldwide survey on the methods, efficacy, and safety of catheter ablation for human atrial fibrillation. Circulation. 2005;111:1100-5.

8. Wokhlu A, Hodge DO, Monahan KH, et al. Long-term outcome of atrial fibrillation ablation: impact and predictors of very late recurrence. J Cardiovasc Electrophysiol. 2010;21:1071-8.

9. Medi C, Sparks PB, Morton JB, et al. Pulmonary vein antral isolation for paroxysmal atrial fibrillation: results from long-term follow-up. J Cardiovasc Electrophysiol. 2011;22:137-41.

10. Sorgente A, Tung P, Wylie J, Josephson ME. Six year follow-up after catheter ablation of atrial fibrillation: a palliation more than a true cure. Am J Cardiol. 2012;109:1179-86.
11. Wazni OM, Marrouche NF, Martin DO, et al. Radiofrequency ablation vs. antiarrhythmic drugs as first-line treatment of symptomatic atrial fibrillation: a randomized trial. JAMA. 2005;293 2634-40.

12. Cosedis Nielsen J, Johannessen A, Raatikainen P, et al. Radiofrequency ablation as initial therapy in paroxysmal atrial fibrillation. N Engl J Med. 2012;367:1587-95.

13. Haïssaguerre $M$, Jaïs $P$, Shah DC, Takahashi A, Hocini M, Quiniou $G$, et al. Spontaneous initiation of atrial fibrillation by ectopic beats originating in the pulmonary veins. $\mathrm{N}$ Engl J Med. 1998;339:659-66.

14. Pappone C, Oreto G, Rosanio S, Vicedomini G, Tocchi M, Gugliotta $F$, et al. Atrial electroanatomic remodeling after circumferential radiofrequency pulmonary vein ablation: efficacy of an anatomic approach in a large cohort of patients with atrial fibrillation. Circulation. 2001;104:2539-44.

15. Pappone C, Rosario S, Oreto G, Tocchi M, Gugliotta F, Vicedomini G, et al. Circumferential radiofrequency ablation of pulmonary vein ostia: a new anatomic approach for curing atrial fibrillation. Circulation. 2000;102:2619-28.

16. Arentz T, Weber R, Burkle G, Herrera C, Blum T, Stockinger J, et al. Small or large isolation areas around the pulmonary veins for the treatment of atrial fibrillation? Results from a prospective randomized study. Circulation. 2007;115:3057-63.

17. Ouyang F, Tilz R, Chun J, et al. Long-term results of catheter ablation in paroxysmal atrial fibrillation: lessons from a 5 -year follow-up. Circulation. 2010;122:2368-77.

18. Ranjan R, Kato R, Zviman MM, et al. Gaps in the ablation line as a potential cause of recovery from electrical isolation and their visualization using MRI. Circ Arrhythm Electrophysiol. 2011;4:279-86.

19. Deftereos S, Giannopoulos G, Kossyvakis C, et al. Colchicine for prevention of early atrial fibrillation recurrence after pulmonary vein isolation: a randomized controlled study. J Am Coll Cardiol. 2012;60:1790-6.

20. Tan AY, Chou CC, Zhou S, et al. Electrical connections between left superior pulmonary vein, left atrium, and ligament of Marshall: implications for mechanisms of atrial fibrillation. Am J Physiol Heart Circ Physiol. 2006;290:H312-22.

21. Pappone C, Santinelli V. Tratamiento ablativo de la fibrilación auricular. Rev Esp Cardiol. 2012;65:560-9.

22. Schreieck J, Ndrepepa G, Zrenner B. Radiofrequency ablation of cardiac arrhytmias using a three-dimensional real-time position management and mapping system. Pac Clin Electrophysiol. 2002;25:1699-707.

23. Uribe W, González E, Baranchuk A, et al. Ablation of atrial fibrillation: first single-center latinamerican consecutive series guided by ICE and 3D electroanatomic mapping. Rev Iberoamer Arrit. 2010. doi: 10.5031/v1i2.RIA10110.

24. Negrete AJ, Salcedo GD, Quesada González K, et al. Resultados de la ablación por radiofrecuencia de la fibrilación auricular paroxística mediante la técnica de aislamiento de las venas pulmonares. Rev Electro y Arritmias. 2010;3:102-5.

25. Shurrab M, DiBiase L, Briceno D, et al. Impact of contact force technology on atrial fibrillation ablation. J Am Heart Assoc. 2015;4:e002476.

26. Reddik V, Dukkipati S, Neuzil P, et al. Randomized, Controlled Trial of the Safety and Effectiveness of a Contact Force-Sensing Irrigated Catheter for Ablation of Paroxismal Atrial Fibrillation: Result of the TactiCath Contact Force Ablation Catheter Study for Atrial Fibrillation (TOCCASTAR) Study. Circulation. 2015; 132:907-15. 